\title{
Ubiquitous Containerized Cargo Monitoring System Development based on Wireless Sensor Network Technology
}

\author{
C.M. Yeoh, B.L. Chai, T.H. Kwon, K.O. Yi, T.H. Kim, C.S. Lee, G.H. Kwark, H. Lim
}

Chee-Min Yeoh, Bee-Lie Chai,Hyoteak Lim

Dongseo University, Churye-2-Dong, Sasang-Gu, Busan, 617-716, South Korea E-mail: yeohcm@dit.dongseo.ac.kr, beelie@dit.dongseo.ac.kr, htlim@dongseo.ac.kr

Tae-Hong Kwon, Ki-One Yi, Tae-Hun Kim, Chang-Sup Lee, Gwang-Hoon Kwark Media Device Lab, Dong-A University, 840, Hadan-2-Dong, Saha-Gu, Busan, 604-714, South Korea E-mail: thkwon@dau.ac.kr, koyrim@dau.ac.kr, rider7979@dau.ac.kr, cslee@dau.ac.kr, paxpia@dau.ac.kr

\begin{abstract}
Due to globalization, global trade is strongly growing nowadays. The use of containers has significantly increased and bringing the change on the shape of the world economy. Thus, monitoring every single container is a big challenge for port industries. Furthermore, rapid development in embedded computing systems has led to the emergence of Wireless Sensor Network (WSN) technology which enabled us to envision the intelligent containers. This represents the next evolutionary development in logistics industry to increase the efficiency, productivity, security of containerized cargo shipping. In this paper, we present a comprehensive containerized cargo monitoring system based on WSNs. We incorporated tilt/motion sensor to improve the network convergence time of container networks. Moreover, we periodically switch the nodes into sleeping mode to save energy and extend the lifetime of the network. Based on the technical implementation on a real container vessel, we strongly believed that our design which employed WSN technology is viable to be implemented in container logistics to improve port services and provide safe transport of containerized cargo.
\end{abstract}

Keywords: Containerized cargo monitoring, sensor node, WSN, Low Power Listening

\section{Introduction}

Containerized shipping made the world smaller and the world economy bigger [1]. Since its introduction in 1960s, containers represent the standard unit-load concept for international freight. Nowadays, the world trade is mostly accomplished by the aid of container using different channel of transportations including railway, airway, maritime, truck and others. These have an extraordinarily large impact that changed the shape of global economy. There had estimated in 2008 more than 500 million Twenty-foot Equivalent Unit (TEU) of containers turnover in the world-wide most important container ports, and, compared with ten years ago, only had around 200 million TEU of containers [2].

The global competition process has been the main external driving force for the new challenging era of the port industry [3]. Ports have to redefine their business process, business strategies as well as service characteristics in order to create and sustain competitive advantage. Port authorities have introduced various add-on services such as safe transport, cargos location and status tracking, rapid customs clearance and so on. In addition, several port authorities have incurred fire damage costs about few hundred million dollars due to weak security assessment and poor cargo monitoring. The damage could be reduced if proper freight monitoring 
is deployed and rescue works could be done as quickly as possible. Besides that, a numbers of international security laws have been enforced to detect and take preventive measures against security incidents affecting ships or port facilities. For instance, International Ship and Port Facility Security Code (ISPS), Container Inspection Program (CIP), Container Security Initiative (CSI), Customs-Trade Partnership Against Terrorism (C-TPAT) and so on are a comprehensive set of measures that in response to the perceived threat to ships and port facilities [4-6]. In order to provide a secure transport for containerized cargo which could improves port services at the same time satisfying the international security laws, port authorities should deploy an autonomous cargo monitoring system extensively. However, one of the challenges is container logistics consists of huge number of nodes that need to be monitored. With the old wireless technology, it is not possible to provide coverage in such dense environment. Thus, one of the possible solutions is to adopt Wireless Sensor Networks (WSN) in the containerized shipping for providing an autonomous cargo monitoring system. WSN is formed by a large numbers of nodes which are capable to measure ambient condition related to the environment surrounding the nodes and transform them into electric signal. Each node is able to communicate among each other and collaboratively transmit data through network to a Base Station (BS). The power of WSN lies in the ability to deploy a large number of nodes that could assemble and configure on its own. These advantages make them very promising in wide range of application from military, health, education, commerce and etc.

Sensor nodes life time is limited to the life time of its battery. Sensor nodes may not be charged once their energy is drained, hence the lifetime of the network depends critically on the battery energy. Nodes mainly drain their energy during the communication process rather than in data processing state. In traditional MAC protocol, most of the energy is wasted by idle listening, collision, overhearing and others. Since node does not able to predict when it will receive message from its neighbors, it must keep its radio in receive mode at all the times. Many measurements have shown that idle listening consumes $50 \%$ to $100 \%$ of the energy required for receiving. For example, Stemm and Katz measure that the idle:receive:send ratios are 1:1.05:1.4 [15]. Besides, for collision, a transmitted packet has to be discarding if it is corrupted and then follow on re-transmissions which will increase energy consumption and latency. A new protocol is needed in order to decrease the initial topology of the network, to reduce the waste of energy from the source and extend the lifetime of the network.

The remainder of this paper is organized as follow: Section 2 describes the basic requirements that need to fulfil when deploying WSN in container networks. Section ?? introduces the overview of our proposed containerized cargo monitoring system and methodology for improving network convergence time. Section 4 explains how the proposed solution deploy the low power listening mechanism. Section ?? provides node and antenna localization in the container. Next, Section 6 presents the experiment and is followed by technical implementation in Section 7. Finally, we conclude this paper in Section 8.

\section{Basic Requirements of Container Network}

This section discusses about various requirements that needed to deploy WSNs-based autonomous cargo monitoring system. Following are several basic requirements that needed to be fulfilled in order to perform optimally in autonomous logistics network.

\section{- Robustness and reliability}

The cargo monitoring systems must equip with the ability to work and cope in rough environments. There are numerous of unexpected conditions in logistics network that could affect the entire system. As a result, autonomous cargo monitoring system should 
be robust and reliable enough to continue their task even though system breakdown in the neighborhood.

\section{- Low power consumption}

The main source of energy for sensor nodes is battery power. Energy is one of the limitations in sensor nodes. Sensor node with low power consumption could yield longer serving period without maintenance.

\section{- Minimum human intervention}

In seaport, there are more than 10,000 containerized cargos that need to be tracked in the monitoring system. Port authorities do not afford to present at every single cargo for maintenance or operation. Hence, cargos monitoring system should be autonomous and minimize the need of human intervention.

\section{- Sensor network longevity}

Sensor nodes that run for 9 months from non-rechargeable power source would be preferred for sensor network nowadays. In logistics environment, typically battery life should sustain vary from 9 to 12 months. This is because it is not practical to change battery frequently for every container in such dense environment.

\section{- Self-organization}

Autonomous is one of the main features provided by wireless sensor network technology. In logistics network, containerized cargos have high mobility from time to time. A lot of containers can join and leave the network when necessary. Thus, nodes need to have self-governing and self-reconstructed capabilities in this environment.

\section{- Small monitoring device}

Monitoring devices that deployed in containerized cargos should not be an obstruction for normal cargo operation. Monitoring devices should be small in size and allow cargo operations run as usual. In addition, a small, tough enclosure should be used to protect the sensor nodes from dust or disruption.

\section{System Design}

In general, there are two types of transportations that are involved in the whole process of containerized cargo shipping such as land carriage and maritime carriage. For long distance shipping, maritime carriage is the best choice among other ways of transportation due to its economical cost. In maritime carriage, more than 10,000 containerized cargos are shipped simultaneously in one huge vessel to a designated seaport. When the vessel reaches the seaport, truck is used to transfer containerized cargos to its destination. The whole process of shipping might take few weeks or months to reach its final destination. As a result, the condition and safety of containerized cargos should be closely monitored in this long shipping process. In order to monitor the condition of the containers throughout the shipping process, we designed a comprehensive containerized cargos monitoring system. We adopted Wireless Sensor Networks (WSN) technology into our monitoring devices to form an autonomous logistics network. In our design, three main networking devices involve in the monitoring system to form a network. A basic unit of networking device, namely Container Network Device (N/D) is positioned in every single container in the system. Container N/D is the main source to collect container's condition. Container N/D will be discussed in more detail in the following section. Next, a Coordination Point $(\mathrm{C} / \mathrm{P})$ is placed on few tall pillars of container terminal or the Wing Bridge of vessel to 
communicate with Container N/D as well as to collect the data from Container N/D and then transmit to control room for future processing. In order to link two separate networks, a Container Network Bridge is used in the system. With bridging, traffic from one network is managed and rebroadcasted to adjacent network segments accordingly.

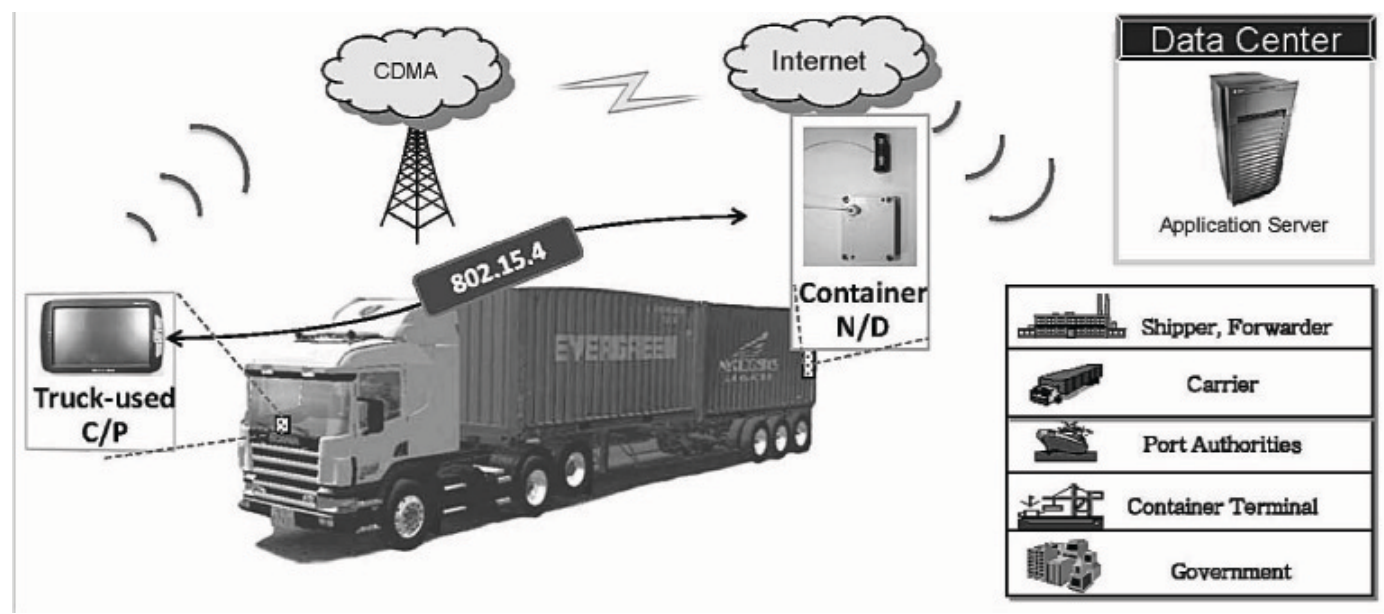

Figure 1: Land Carriage Communication

A secure containerized cargo monitoring system should not stop until seaport. Thus, our cargo monitoring system provides end to end security until cargos final destination such as warehouse or distribution center. We used two core networking devices during the shipment through land carriage as shown in Figure 1. We placed a truck-used Coordination Point in front of the driver seat to communicate with the Container N/D inside the container that is being transported by the truck. Container N/D sends the sensing data to the truck-used $\mathrm{C} / \mathrm{P}$ with IEEE 802.15.4 complied interface [8]. Through the truck-used $\mathrm{C} / \mathrm{P}$, truck driver is able to discover the containers information such as identity of container, Container N/Ds id, temperature and humidity of the container and so on. The truck-used $\mathrm{C} / \mathrm{P}$ is equipped with Code Division Multiple Access (CDMA) modem to forward the acquired data to the main server through CDMA network for further processing. As a result, the main server will obtained updated data at anytime and at anywhere from the moving truck.

\subsection{Container Network Device}

Container Network Device (N/D) is the basic unit of networking device which is placed in every single container in the system. The main responsibility of $\mathrm{N} / \mathrm{D}$ is to sense the containers condition and transmit those sensing data to Coordination Point $(\mathrm{C} / \mathrm{P})$. N/D is capable to form a Multi-hop Cluster Tree network automatically among N/Ds and collaboratively pass on the data to $\mathrm{C} / \mathrm{P}$ when the $\mathrm{N} / \mathrm{D}$ is situated out of radio range of the $\mathrm{C} / \mathrm{P}$. Each cluster has one parent node to coordinate the nodes in the cluster and forward its cluster nodes data to $\mathrm{C} / \mathrm{P}$. Like any other commercial sensor nodes such as TelosB, MicaZ and etc $[9,10], \mathrm{N} / \mathrm{D}$ is powered by two AA batteries and has limited resources in term of computational capability, storage and so on. Thus, low power listening mechanism is deploy onto the N/D which will be discussed in detail in next section. Additionally, application and tasks for N/D are designed with low resource consumption and efficiency in mind to prolong the N/Ds lifetime.

Table 1 shows the basic components of Container N/D. We employed TinyOS 2.1 which is the latest up-to-date version of component based operating system targeting for wireless sensor network in our N/D [11]. This version has improved various features of TinyOS version 1.0 and 
Table 1: Basic Components of Container N/D

\begin{tabular}{ll}
\hline Parameter & Values \\
\hline MCU & MSP430 Family \\
Operating System & TinyOS 2.1 \\
Sensor & Tilt Sensor SA1 \\
& Temperature/Humidity \\
& 3.5 volt \\
\hline
\end{tabular}

supported low power CC2420 RF transceiver extensively which is required in our design. Besides temperature/humidity sensor, we incorporated tilt sensor into N/D. Tilt sensor is used to detect the motion of the container which will be discussed in detail in Section ??

\subsection{Coordination Point}

The main function of Coordination Point $(\mathrm{C} / \mathrm{P})$ is to communicate with NDs as Base Station and forwards the data from $\mathrm{N} / \mathrm{Ds}$ to main server for further processing. Thus, $\mathrm{C} / \mathrm{P}$ is equipped with wireless interface complies with IEEE 802.15.4 standard to communicate with N/Ds as well as internet connectivity via Ethernet or CDMA network to transmit the acquired data to the application server. Unlike $\mathrm{N} / \mathrm{D}, \mathrm{C} / \mathrm{P}$ is powered by unlimited power supply and has superior processor and storage. Generally, there are two types of $\mathrm{C} / \mathrm{Ps}$ that are used in our system which are seaport-used $\mathrm{C} / \mathrm{P}$ and truck-used $\mathrm{C} / \mathrm{P}$. The differences between these two $\mathrm{C} / \mathrm{Ps}$ are antenna type, radios range, touch screen option, and so on. The seaport-used $\mathrm{C} / \mathrm{P}$ which is placed on a tall pillar of container terminal or on Wing Bridge of container vessel. It has higher-end of antenna which could provide wider coverage if compared to truck-used $\mathrm{C} / \mathrm{P}$. On the other hand, truck-used $\mathrm{C} / \mathrm{P}$ which is used by land carriage and is placed in front of drivers seat. This $\mathrm{C} / \mathrm{P}$ has an interactive 7 -inch touch screen that could provide the necessary container's information such as container identity number, N/Ds id, temperature and humidity of the container that being transported by the truck. This $\mathrm{C} / \mathrm{P}$ uses CDMA interface to forward the acquired data from N/D to application server for further processing. As a result, user could get the containers condition at any time and at anywhere during the shipping.

\subsection{Nodes Motion Detection for Improving Network Convergence Time}

In the beginning of this paper, we have discussed one of the essential basic requirements to form a logistics network with WSNs is self-organization. Especially in logistics network, thousand of containerized cargos join and leave the network in a short period. Thus, nodes that attached to containers should be self-aware and react according to the current state of the environment. However, it is not practical for nodes to wait for triggering from Status Timer then only broadcast the topology changes when a node is leaving. This is because some nodes might mistakenly send packet to the leaving node as that node still exist in the nodes neighbor table. As a result, real time motion detection is needed for improving network topology reconstruction time when there is a node leaving the network.

As a result, we proposed to incorporate tilt/motion sensor into Container N/D to detect the movement of the container. The tiny tilt sensor or tilt switch has only $3 \mathrm{~mm} \times 8 \mathrm{~mm}$ in size [12]. This sensor has high sensitivity and consumes ultra low power ranging from 0.00025 to $5 \mathrm{~mA}$ which is suitable for our application. 


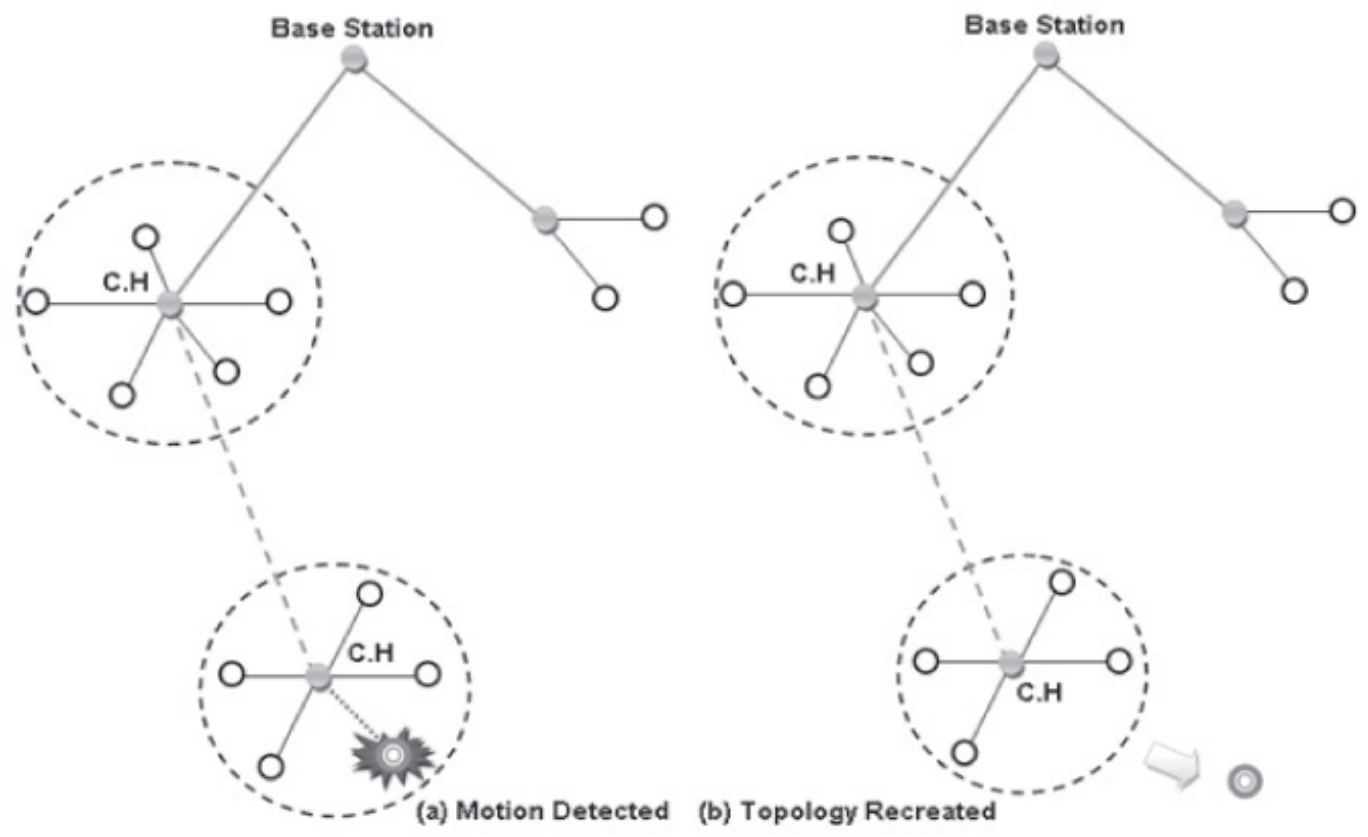

Figure 2: Node Leaving Network Scenario

The main responsibility of the tilt sensor is to detect any movement of the container. If a container is moved from one place to another place, the movement will trigger its tilt state as demonstrated in Figure 2. Once the movement is detected by tilt sensor, N/D immediately broadcasts the leave packet to its 1-hop neighbor. Then, all nodes within 1-hop of the leaving N/D remove its relationship with the leaving N/D. While parent node of the leaving node forwards the leave packet to its parent until the information reaches base station. As a result, the network topology has been reconstructed rapidly without any human intervention. The performance of employing motion sensor into N/D for improving convergence time is presented in Section ??.

\section{Low Power Listening Mechanism}

In most of the protocol designed for wireless networks had a common problem, which was the receiver must always be on. In most of the cases that the power consumed during listening to an idle channel same as power consumed when receiving data. This idle listening method was very power-inefficient in wireless sensor network [14]. Moreover, numerous of wireless sensor network researchers found that energy saving can be achieved by duty cycling. Node designed with low duty-cycle MAC protocol should periodically switch its radio interface to active and sleep. The nodes operating time was divided into active-sleep cycles. Therefore, in each cycle consisted of two durations: active and sleep. Node regular transmissions and receptions in active duration, while in sleep duration the node was power off its radio interface and reduce the energy consumption.

In proposed solution, we apply low power listening (LPL) mechanism on N/D but do not apply on $\mathrm{C} / \mathrm{P}$. Given that $\mathrm{C} / \mathrm{P}$ has installed with unlimited power supply, we can utilize this advantage to improve the network performance. As a result, $\mathrm{C} / \mathrm{P}$ is always in idle listening mode and never switches to sleep mode when it's not transmitting or receiving message. In contrast, $\mathrm{N} / \mathrm{D}$ is powered by two AA batteries and low power listening mechanism should apply on the N/D to reduce the power consumption and longevity of service. C/P does not apply LPL mechanism, 


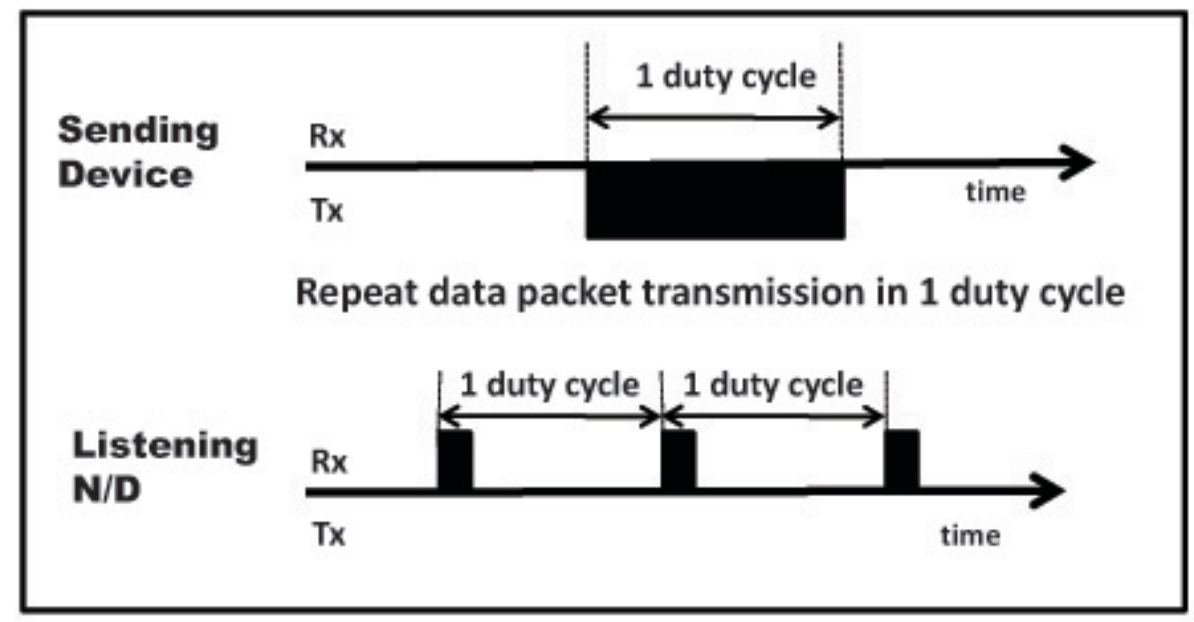

Figure 3: N/D Low Power Listening Model

$\mathrm{C} / \mathrm{P}$ turns on its radio interface all the time. While $\mathrm{N} /$ Ds operating time is divided into low duty cycles as shown in the Figure 3. In each duty cycle, divided into active-sleep intervals, then each active-sleep interval consists of two durations: active and sleep. Active duration is for node turns on its radio interface and sniffing for transmitting data, while in sleep duration the node will power off its radio interface to reduce energy consumptions.

With the low power listening mechanism, when any device in the network would like to send message to any device in the topology, the devices have to consider the role of the receiver. If the receiver is $\mathrm{C} / \mathrm{P}$, the sender requires send a single packet as usual is sufficient. However, if the receiver is $\mathrm{N} / \mathrm{D}$, the sender has to send the same message repeatedly in one duty cycle similar in Figure 3. If sender would like to broadcast a message or the sender do not know receiver role, sender has to send the message in one duty cycle too.

\section{Node and Antenna Localization}

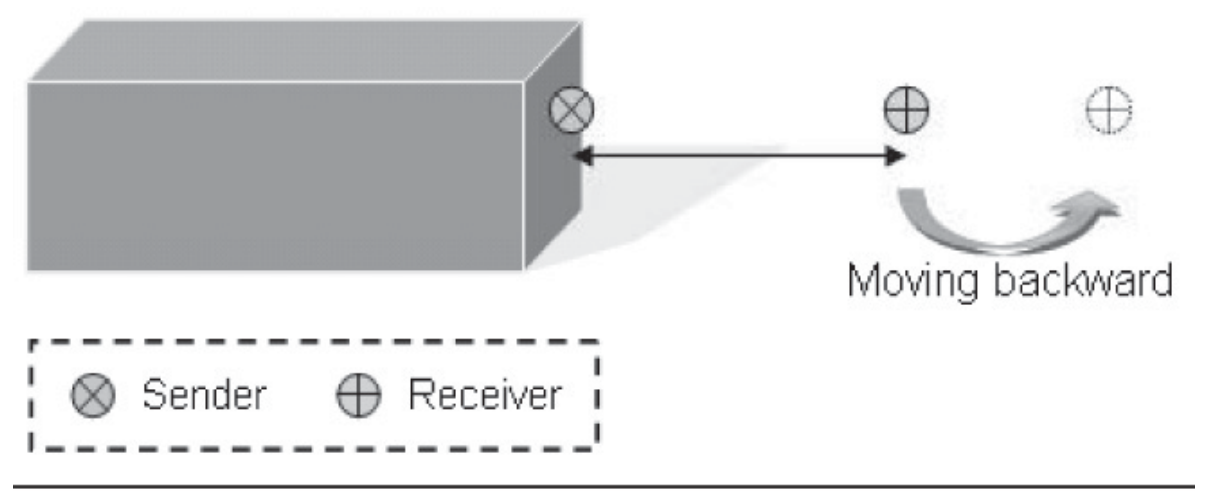

Figure 4: Test Methodology

The placement of node and its antenna is a vital issue in container environment. This is because improper node placement will cause poor performance of data transmission and might affect normal cargo operation.

We proposed and tested with various positions of $\mathrm{N} /$ Ds antenna in the container to ensure optimum performance. The performance matrix of this test is measured by the maximum dis- 
Table 2: Result of various node placements

\begin{tabular}{cc}
\hline Parameter & Maximum Distance $(\mathbf{m})$ \\
\hline A & 14 \\
B & 25 \\
C & 35 \\
\hline
\end{tabular}

tance that two container N/Ds could stay connected. In this test, one container N/D acts as sender and another container N/D operates as receiver as illustrated in Figure 4. The sender is positioned statically in the container and keeps sending data every 0.5 second to the receiver. Receiver keeps moving apart from the sender until before the receiver could not receive any data packet from the sender. Then, the distance between sender and receiver is measured. All tests were conducted with containers door closed. We used an external PCB antenna with $2 \mathrm{dBi}$ and container $\mathrm{N} / \mathrm{D}$ with $\mathrm{RF}$ power about $-2 \mathrm{dBm}$ in the test. In placement $\mathrm{A}$, we attached the container N/D inside the container upper right and the antenna is placed at container's door ledge as illustrated in Figure 5a. In placement B, container N/D is positioned inside the container upper center as depicted in Figure 5b. Next, in placement C, we attached the container N/D antenna outside container at the hinge while the container $\mathrm{N} / \mathrm{D}$ seated inside the container to obtain the temperature/humidity of the container as demonstrated in Figure 5c.

Referring to Table 2, placement $\mathrm{C}$ which attaching the container $\mathrm{N} / \mathrm{Ds}$ antenna at the hinge yields great reception if compared to other placements. Besides providing best performance, placement $\mathrm{C}$ also provides protection on antenna from human interrupt or any undesired damages. As a result, placement $\mathrm{C}$ was selected as an ideal place to setup container N/D and its antenna in the container.

\section{Experiment and Analysis}

\subsection{Network Topology Reconstruction Time}

In this section, we evaluate the performance of using motion sensor on $\mathrm{N} / \mathrm{D}$ that could improve network topology reconstruction time.

We carried out the experiment by using Qualnet version 4.5.1 [13]. Qualnet is the commercial successor to Glomosim which has a widespread set of high fidelity models ranging from the physical layer to application layer that allows modeling a variety of emulated networks. Qualnet's scenario designer provides easy topology configuration while it's graphical Analyzer serves well for performance analysis of the emulated network. Besides that, latest version of Qualnet supports sensor networks library that includes MAC and PHY layer with IEEE 802.15.4 standard compliance which are well-fitted in our system. As discussed previously, short network topology recreation time is favorable in container networks. Fail to update the network topology in a short period of time will cause unwanted broken link and data loss. A state of convergence is achieved once all routing information has been distributed to all nodes participating in the routing protocol process. In this simulation, we use convergence time as our performance metrics which measures how fast a group of nodes could reach the state of convergence.

Our simulation consists of 17 nodes across a plain area of 500x500 meters as shown in Figure 6. Node 1 acts as the Coordination Point (C/P) or base station and located at position (141.1, 487.74). Node 6 and Node 8 are the only nodes in the coverage of $\mathrm{C} / \mathrm{Ps}$ radio range. Thus, these two nodes have 1 hop distance from $\mathrm{C} / \mathrm{P}$ while the rest of the nodes are required to form multi- 


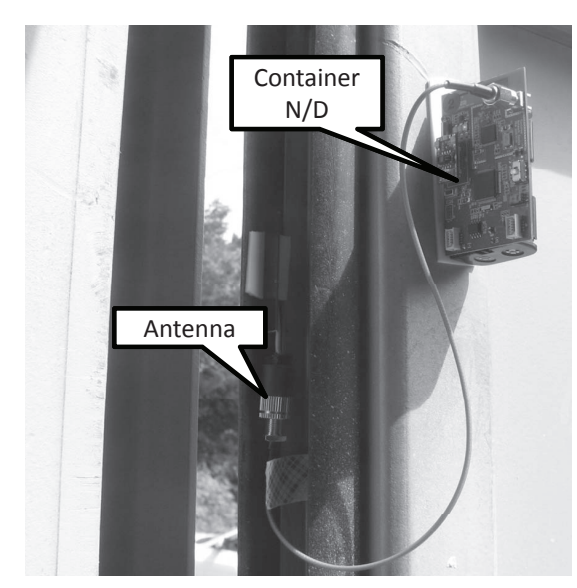

(a) Placement A

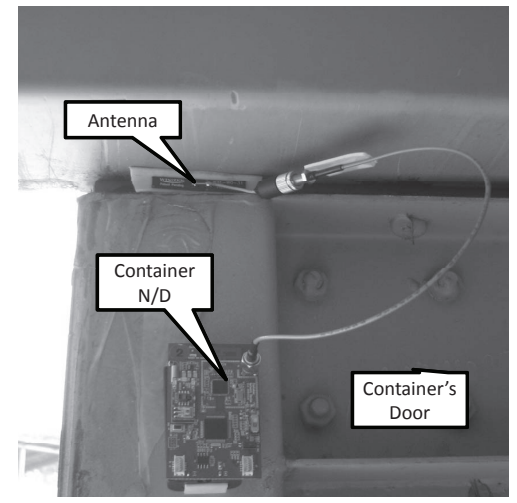

(b) Placement B

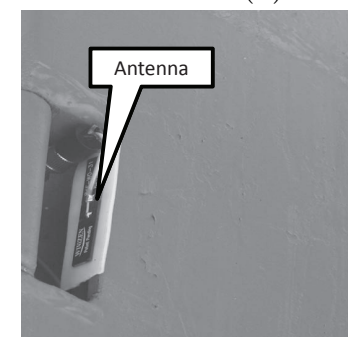

(c) Placement C

Figure 5: Experiement Placement

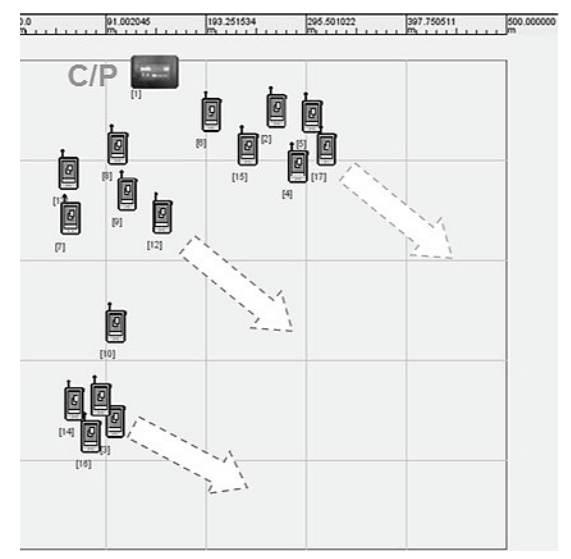

Figure 6: N/D Motion Test Scenario

hop network in order to reach $\mathrm{C} / \mathrm{P}$. Once all nodes have joined the parent node and formed a converged network, node 3, node 12 and node 17 leave the network and trigger its virtual motion sensor consecutively. In this simulation, we assumed that whenever node changed its position, the motion sensor is triggered.

Figure 7 shows the simulation result of using tilt detection and without using tilt detection in our system. The result shows that by using the motion sensor in routing portion, the convergence time has improved significantly even though with diverse of status time interval. Averagely, about 22.76 seconds after the node leaved, the network has back to convergence state. On the other hand, the convergence time of nodes without using motion sensor is performed poorly and is with respect to status time interval. This is because the parent node needs to wait for triggering from its status timer then only broadcasts the topology changes after the node has leaved. Thus, the 


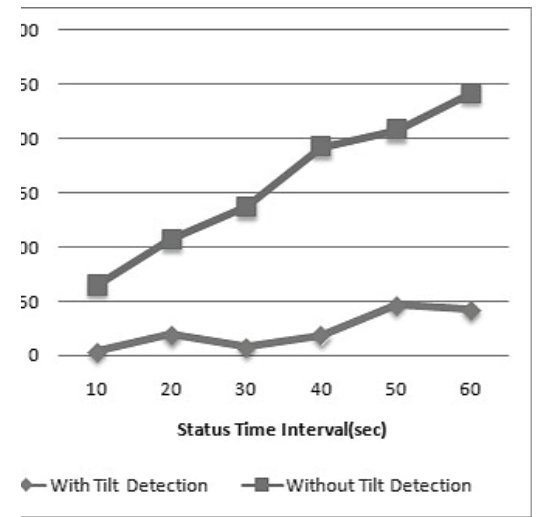

Figure 7: Effect of Using Motion Sensor on Convergence Time

larger the status time interval, nodes take much longer time to reconstruct the network topology. In a nut shell, incorporating motion sensor in our system for enabling real time leave network functionality greatly improved the convergence time of the network which is essential in container networks.

\subsection{Evaluation on Energy Consumption}

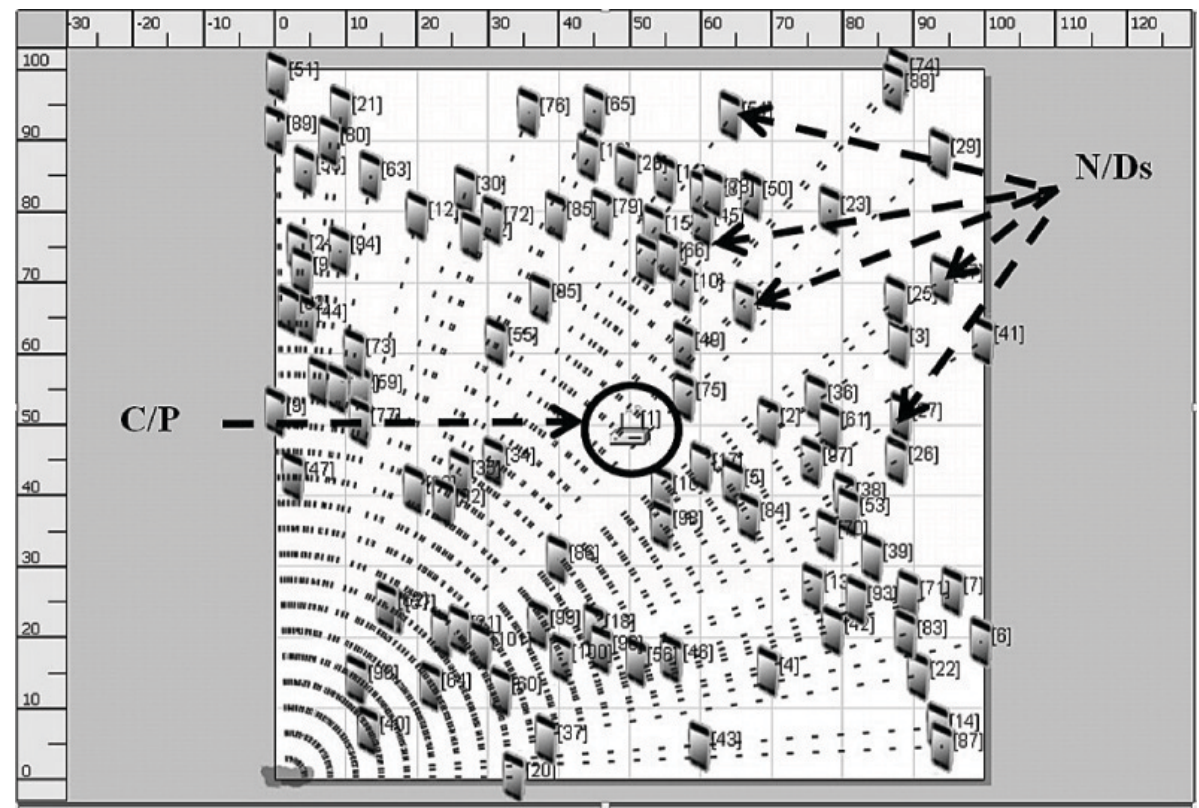

Figure 8: 100 of Static N/Ds Test Scenario

For comparison of the energy efficient of the proposed solution, we carry out the experiment by using Qualnet version 5.0.2 [13]. We implement two types of N/D in Qualnet network simulator: $\mathrm{N} / \mathrm{D}$ with low power listening mechanism and N/D without low power listening mechanism. $\mathrm{N} / \mathrm{D}$ with low power listening, the message exchange is implemented as descript in the Section 4. On the other hand, the implementation of N/D without low power listening mechanism, N/D never switch off its radio interface, therefore either unicast or broadcast message in the network always send in single message as usual but not in long one duty cycle. The simulation is running in twenty-four hours with $100 \mathrm{~N} /$ Ds and single $\mathrm{C} / \mathrm{P}$ are created in an open area and $\mathrm{C} / \mathrm{P}$ at 
the middle of the test area as show in Figure 8. The simulation configuration summarizes in Table 3. In the Figure 9 depicts the cumulative of the total energy consumption on 100 of N/DS

Table 3: Simulation Parameter

\begin{tabular}{ll}
\hline Variable & Values \\
\hline Duration for 1 duty cycle & 1 second \\
Radio on duration for each duty cycle & 0.01 second \\
Transmitting/Receiving/ Idle Listening Circuitry Power Consumption & $70.0 \mathrm{mWatt}$ \\
Sleep Circuitry Consumption & $0.035 \mathrm{mWatt}$ \\
Power Amplifier Inefficiency Factor & 6.5 \\
Supply Voltage (volt) & 3.5 volt \\
\hline
\end{tabular}

respectively between N/Ds with LPL mechanism and N/Ds without LPL mechanism. Since the $\mathrm{C} / \mathrm{P}$ never switch off its radio interfaces as other $\mathrm{N} / \mathrm{Ds}$ in both simulation testing, the total energy consumption in both scenarios are almost same. On the other hand, the total energy consumption for 100 of N/Ds deploy with LPL mechanism have dramatically reduce the drain of energy. In the twenty-four hours simulation test has including the energy for building topology in the first hour of the simulation and following hours are working on topology maintenance. During topology maintenance, each N/D will broadcast its current status message to its neighbor every second, especially to its direct parent node and child nodes. According to the test result shows in the Figure 9, after twenty-four hours execution the N/Ds applied LPL mechanism total energy consumption for all $100 \mathrm{~N} / \mathrm{Ds}$ in the network have consumed 4 Joule and average energy consumption per hour is 1.7 Joule. However, the total energy consumption in the $100 \mathrm{~N} /$ Ds does not apply LPL mechanism is 165 Joule and average energy consumption per hour is 7 Joule. It has saved up to $97 \%$ of the total of network energy consumption with apply LPL mechanism in the N/Ds. LPL mechanism reduced the drain of energy dramatically.

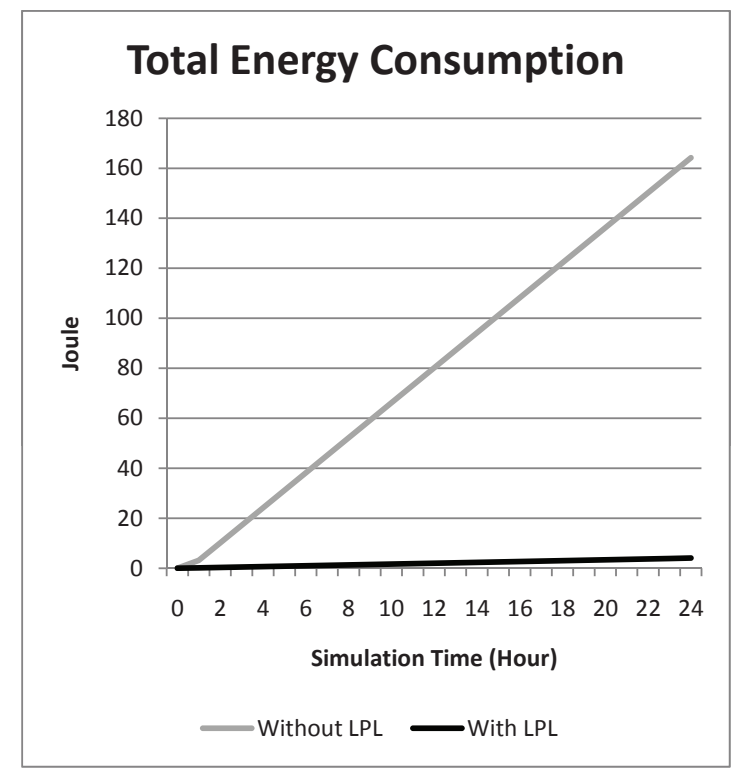

Figure 9: Cumulative of 100 N/Ds Total Energy Consumption 


\section{Technical Implementation}

In order to further study our monitoring system and analyze the feasibility of our proposed system in real logistics environment, we implemented our wireless sensor networks based cargo monitoring system on a real container vessel named Hyundai Jakarta. The vessel has the capability to ship 6800 TEU of containers at a time and its specification is shown in Table 4. This test was carried out to monitor dangerous goods in the vessel during the shipping from Busan, South Korea to Antwerp, Belgium from 26th January 2009 to 20th February 2009. In this

Table 4: Vessel's Specification

\begin{tabular}{ccccc}
\hline Length(m) & Width(m) & Depth(m) & Total Ton(G/T) & Load (TEU) \\
\hline 294 & 40 & 20.17 & 75000 & 6800 \\
\hline
\end{tabular}

test, we installed 10 container N/Ds and 2 seaport-used C/Ps in the vessel. We placed each $\mathrm{C} / \mathrm{P}$ at the Wing Bridge of the vessel to receive the data from container N/Ds as shown in Figure 10a. On the other hand, 10 dangerous good containers were mounted with Container N/D to monitor the condition inside the container. Figure 10b depicts the position of N/D inside the container. In order to communicate with $\mathrm{C} / \mathrm{P}$ with closed door, antenna of $\mathrm{N} / \mathrm{D}$ must not be positioned inside the container. Based on our test on Section ??, mounting the container N/Ds antenna at the hinge could yield great reception as shown in Figure 10c. Besides providing best performance, this placement also provides protection on antenna from human interrupt or any undesired damages.

We carried out several tests on the vessel during voyage. Firstly, we tested the communication between $\mathrm{C} / \mathrm{P}$ and N/D in line-of-sight (LOS) condition by sending data periodically with 0.5 second interval. Secondly, we tested the reliability of our Multi-Hop routing protocol on the vessel. Figure 10d shows the placement of N/Ds where N/D 2 was out of C/Ps radio range which was in Non line-of-sight (NLOS) condition while N/D 1 had LOS with C/P as shown in Figure 10e. The tests show promising result as we successfully received more than $93 \%$ of data packets from N/Ds for both tests.

The test was proceeded to verify the reliability of sensing data transmission for few days. In this test, we configured N/Ds to obtain containers temperature and humidity for every hour. A unique node ID was mapped with container identity number accordingly before the test. We logged the data received from container N/Ds for further processing as shown in Table 5.

Table 5: Sample of Logged Data

\begin{tabular}{ccccc}
\hline node ID & container ID & Temp & Hum. & Time \\
\hline 9 & HDMU2286533 & 3 & 70 & $2009-01-26,00: 16: 21$ \\
2 & HDMU2450610 & 3 & 72 & $2009-01-26,00: 20: 40$ \\
7 & HDMU2179849 & 5 & 70 & $2009-01-26,00: 23: 24$ \\
2 & HDMU2450610 & 3 & 72 & $2009-01-26,01: 19: 16$ \\
7 & HDMU2179849 & 5 & 69 & $2009-01-26,01: 22: 00$ \\
\hline
\end{tabular}

Throughout the experiment, we successfully obtained important data such as temperature, humidity, motion and so forth on the ocean environment. Besides that, we also have better understanding of logistics operation flow and concrete concept of container stacking scenario on a 


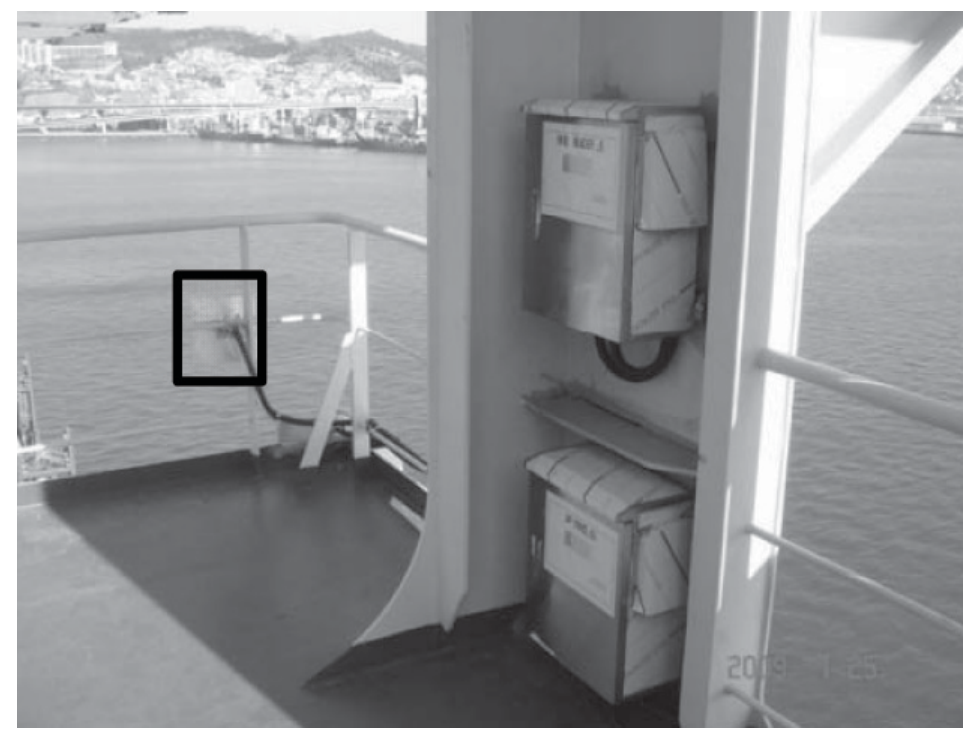

(a) Installation of CP on Wing Bridge

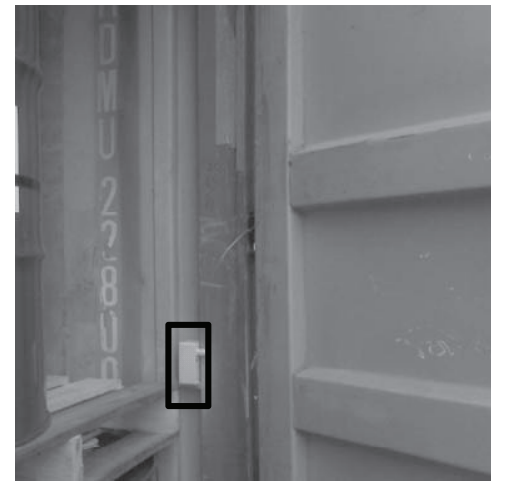

(b) Installation of $\mathrm{N} / \mathrm{D}$ inside Container

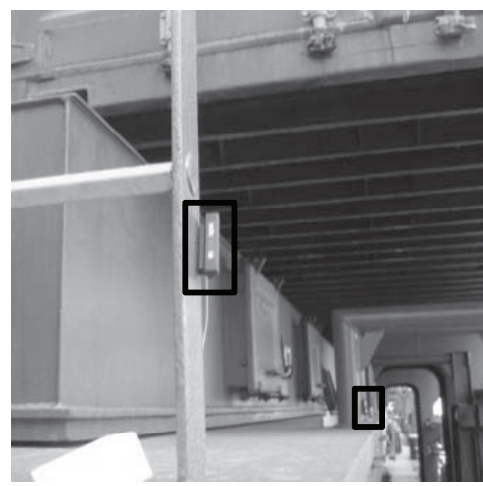

(d) Installation of $\mathrm{N} / \mathrm{D}$ for Multi-Hop Test

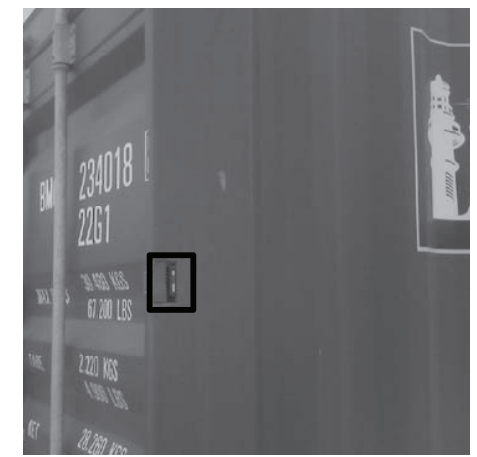

(c) Installation of N/D's Antenna

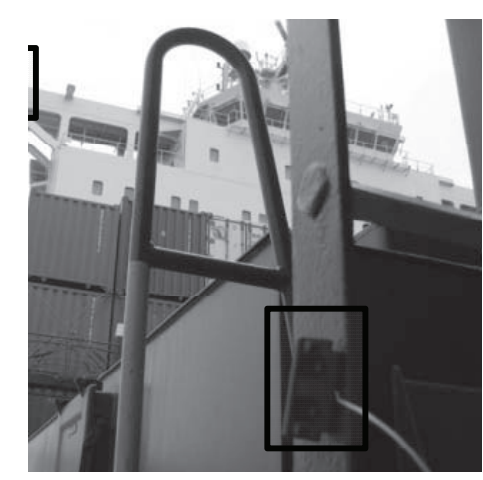

(e) $\mathrm{C} / \mathrm{P}$ and $\mathrm{N} / \mathrm{D}$ with LOS Condition

Figure 10: Installation of $\mathrm{C} / \mathrm{P}$ and $\mathrm{N} / \mathrm{D}$

container vessel. Furthermore, we also have experienced in devices installation and testing during boarding and voyage. As a result, the feasibility of data gathering and container monitoring through wireless sensor networks technology on a container vessel has been studied and verified. 


\section{Conclusions}

This paper addressed the deployment of Wireless Sensor Network in transport and logistics industry. We identified and discussed the basic requirements that are needed to form an autonomous cargo monitoring system with WSN. Network convergence time is one of the important attributes especially in container networks. Therefore, we incorporated tilt/motion sensor to improve the topology re-construction time by immediately broadcast leave message after the containers movement is detected by the sensor. Moreover, we deployed low power listening mechanism into the container communication devices to reduce the energy consumption in the network. Besides that, node placement is an important issue to obtain optimum performance. We conducted the test which positioned the NDs antenna at various places in the container. From the result, we discovered that the antenna at the containers hinge yield the best result in our tests. On the other hand, we also implemented our system on real container vessel. From the result, we strongly believed that our proposed solution is feasible for container networks. Through the test, we have better understanding of logistics operation flow and concrete concept of container stacking scenario on a container vessel that would be useful in the future.

\section{Acknowledgments}

This work was supported by the grant No. B0009720 from the Regional Technology Innovation Program of the Ministry of Knowledge Economy(MKE). Corresponding author is GwangHoon Kwark.

\section{Bibliography}

[1] M. Levinson, The Box: How the Shipping Container Made the World Smaller and the World Economy Bigger, Princeton University Press, 2006

[2] Container throughput in the world-wide most important container ports, http://www. hafen-hamburg.de/content/view/33/33/lang, en/

[3] Chlomoudis, C.I. and Pallis, A.A., Port Governance and the Smart Port Authority: Key Issues for the Reinforcement of Quality Services in European Ports, Proc. of the 10th World Conference on Transport Research, (CDRom), Istanbul, June, 2004

[4] Busan Regional Maritime Affaire and Port Office: http://www . portbusan.go.kr/Service . do?id=en_sub4 -1 ,

[5] CSI in Brief: http://www.cbp.gov/xp/cgov/trade/cargo_security/csi/csi_in_brief . xml

[6] C-TPAT: Customs-Trade Partnership Against Terrorism: http://www.cbp.gov/xp/cgov/ trade/cargo_security/ctpat

[7] A. Mainwaring, J. Polastre, R. Szewczyk, D. Culler, J. Anderson, Wireless sensor networks for habitat monitoring, ACM Int. Workshop on Wireless Sensor Networks and Applications (WSNA'02), Atlanta, GA, September 2002.

[8] IEEE 802.15.4 WPAN-LR Task Group: http://www.ieee802.org/15/pub/TG4.html

[9] TelosB Datasheet: http://www.xbow.com/Products/Product_pdf_files/Wireless_pdf/ TelosB_Datasheet.pdf 
[10] MicaZ Datasheet: http://www.xbow.com/Products/Product_pdf_files/Wireless_pdf/ MICAz_Datasheet.pdf

[11] TinyOS: http://www.tinyos.net/

[12] Tilt Switch: http://www.das-co.com/bbs_form/Fckeditor/upload/TILT_SW_TS3.pdf

[13] Scalable Network Technologies. Qualnet: http://www.scalable-networks.com

[14] El-Hoiydi A, Aloha with preamble sampling for sporadic traffic in ad-hoc wireless sensor networks, Proc. of IEEE Int. Conference on Communications, NY, USA, 5:3418-3423, 2002

[15] M. Stemm and R. H. Katz, Measuring and reducing energy consumption of network interfaces in hand-held devices, IEICE Trans. on Communications, E80-B(8): 1125-1131, 1997. 